\title{
Culture, mondialisation et médiatisation
}

Modernités parallèles au Mexique

\section{Kristina Tiedje}

\section{OpenEdition}

\section{Journals}

Édition électronique

URL : https://journals.openedition.org/pa/1878

DOI : $10.4000 /$ pa. 1878

ISSN : 2273-0362

Éditeur

Université Lumière Lyon 2

\section{Édition imprimée}

Date de publication : 1 janvier 2005

Pagination : 53-58

ISBN : 1634-7706

ISSN : 1634-7706

\section{Référence électronique}

Kristina Tiedje, «Culture, mondialisation et médiatisation », Parcours anthropologiques [En ligne], 5 | 2005, mis en ligne le 06 juillet 2021, consulté le 21 juillet 2021. URL : http://journals.openedition.org/ pa/1878 ; DOI : https://doi.org/10.4000/pa.1878 


\section{CULTURE, MONDIALISATION ET MÉDIATISATION : MODERNITÉS PARALLÈLES AU MEXIQUE}

Un changement non négligeable s'est produit dans un petit village de la région de la Huastèque au Mexique central où je travaille depuis environ dix ans : l'installation de l'électricité dans cette communauté nahua longuement classifiée comme étant marginalisée selon les enquêtes nationales, faute de route, d'électricité et d'eau potable. Après de longues années de mobilisation, la population, qui s'était organisée pour réclamer ces services au gouvernement municipal, a enfin réussi à les obtenir. Dès lors, d'autres changements se mettent en place.

Lors de mon retour sur le terrain en 1999 après un an d'absence, le village ne semble pas avoir changé : la journée se déroule tranquillement, comme avant. Toutefois, à la tombée de la nuit autrefois un moment privilégié pour discuter en famille et pour raconter les histoires des ancêtres aux petits avant d'aller se coucher - un nouveau mouvement se met en place. À ma surprise, plusieurs voisines arrivent pour rendre visite à ma famille d'accueil. L'hôte apporte des chaises pour les invitées et les place au milieu de la pièce à la manière traditionnelle. Je pars dans la cuisine pour préparer le café avec la maîtresse de maison. En arrivant dans la pièce avec le café, je remarque que la conversation a cessé et que tous les membres de la famille, la grand-mère et l'enfant de trois ans inclus, ainsi que les invitées, ont dirigé leur attention sur un immense téléviseur, que je remarque pour la première fois. Je distribue le café avec la maîtresse de maison, Florencia, et je m'assieds enfin sur une chaise qui m'a été assignée. Ana, la petite fille de six ans se met sur mes genoux en chantant la musique de la telenovela (feuilleton), affichée sur l'écran ${ }^{1}$. La protagoniste de l'épisode du jour est une femme riche de la ville de Mexico. Elle est plutôt mince et musclée, aux cheveux courts décolorés en blond platine, elle fume des cigarettes, génère des intrigues, et porte des pantalons très serrés et des jupes très courtes. Pendant toute la séance, le public (bilingue en nahuatl et en espagnol) reste muet devant la télé. Ana m'explique plus tard que la protagoniste "vit toujours des histoires d'amour avec des hommes puissants et très beaux » (j'apprends aussi qu'ils sont « beaux » car ils sont grands, blonds aux yeux bleus). Après la séance, les voisines se souhaitent une bonne nuit et tout le monde part se coucher. La scène se reproduit le lendemain, le jour d'après et tous les soirs de la semaine.

Je commence avec cette note de terrain, car elle nous apporte une impression sur les mondes rapprochés par la mondialisation de l'image et par la présence des médias dans la vie des ruraux qui vivent en province. Le village de Florencia se trouve et à l'écart des grands centres urbains du Mexique central et dans un pays qui est parfois classifié comme faisant partie des "pays en voie de développement ». Cette communauté est située dans la zone rurale montagneuse de la Sierra Madre Orientale, dans la région de la Huastèque, dans laquelle quatre-vingt pour cent de la population vit dans des communautés rurales loin des chefs-lieux (cabeceras municipales), et environ soixante pour cent parle une langue indigène. L'avènement de l'infrastructure, qui reste pourtant très basique avec une route rurale et l'électricité, a produit des situations nouvelles auxquelles se confronte l'anthropologue; un monde qui semble tout autre que le monde des peuples "lointains" qui a donné lieu à la formation de notre discipline. Comme le montre cet exemple, nous sommes alors à la rencontre de personnes dont la vie de tous les jours est bousculée par des changements structurels engendrés par les forces de la mondialisation. Il s'agit alors d'un monde rapproché, car on peut appréhender la mondialisation comme "un processus qui génère des espaces contradictoires, caractérisés par la contestation, la différenciation interne, et les croisements continus des frontières »(Sassen 1998 : xxxiv). Au Mexique, ce rapprochement se produit par l'invasion culturelle de la télévision, par le mouvement de personnes par la migration temporaire vers les centres urbains ou transfrontalière (aux ÉtatsUnis), et par le tourisme.

Une grande majorité des personnes nahuas de la région de la Huastèque vit sur la terre de leurs ancêtres, si ce n'est qu'ils ont pu le récupérer suite à la réforme agraire post-révolutionnaire sous forme de terres communales (ejido ou communauté indigène). La Réforme Agraire (Reforma Agraria) qui date de 1917, visait à apaiser les masses rurales après la Révolution Mexicaine. La réforme avait créé un cadre légal pour restituer les terres expropriées à la population rurale, composée de personnes d'origine indigène qui avaient perdu leurs terres durant la colonisation et l'époque du libéralisme économique après l'Indépendance (voir De Janvry 1996, Stephen 2002, Tiedje 2002).

À présent, les ruines des pyramides, situées en haute montagne sont utilisées pour faire pousser les caféiers, le café étant la culture destinée à la vente dans cette région à un taux de marginalisation élevé. En revanche, les grandes pyramides des anciennes Cités de la civilisation huastèque, El Consuelo et Tamtok, situées dans la vallée, ont suscité l'intérêt des archéologues français, mexicains, et américains, et après des années de fouilles et de reconstruction, servent d'attractions touristiques, ayant été déclarées «patrimoine de la région» par l'Institut National d'Histoire et d'Archéologie (INAH) ${ }^{2}$.

L'industrie du tourisme national et international s'appuie en grande partie sur les attractions du 
patrimoine naturel et culturel du pays, et apporte environ dix pour cent au PIB national (Sectur 2004). Pendant que les touristes s'informent de la culture préhispanique du Mexique, les adolescents issus de zones rurales s'amusent à jouer au basket-ball et au football, en imitant leurs héros de la télévision. Le samedi soir pour aller au bal ou le dimanche pour aller au marché du chef-lieu, les jeunes filles échangent leurs jupes traditionnelles et leurs uniformes d'école avec une tenue plus sexy, se maquillant à la façon des filles dans les telenovelas en jean avec des T-shirts serrés et transparents. Les jeunes enfants, souvent encore unilingues en Nahuatl, leur langue maternelle, s'approprient l'espagnol, la langue de la maestra, de l'institutrice, en regardant les dessins animés à la télévision tout en en faisant leurs devoirs.

Une question s'impose quant à la démarche sur le terrain de l'anthropologue qui est désormais confronté à un mélange de représentations qui prennent forme dans les discours de nos interlocuteurs influencés par la présence inéluctable des médias et de la télévision dans leur vie quotidienne. Ces changements soulèvent par ailleurs d'autres questions qui concernent entre autres le rôle des médias et de la «culture de la télévision" dans le façonnement de l'imaginaire national en relation avec les forces de la mondialisation. Les exemples montrent que la télévision a pris une place décisive dans la vie quotidienne des ruraux et des peuples indigènes au Mexique et remet en question l'ancienne dichotomie entre le Mexique "profond» et « oublié » et le Mexique «imaginé » et " moderne » (Bonfil 1987). Alors que le Mexique était marqué pendant longtemps par une "géographie de la modernité " dans l'imaginaire national, séparant les zones rurales (traditionnelles) des zones urbaines (modernes) et faisant du Mexique rural un Mexique silencieux et muet faute de représentation dans les médias (Lomnitz 2001), l'appropriation des images par les peuples indigènes et les ruraux s'engagent dans la création fluide d'imaginaires alternatifs.

\section{Modernités parallèles}

La puissance de l'anthropologie contemporaine se situe dans sa préoccupation actuelle à comprendre les pratiques et les systèmes de représentation des personnes dans des contextes historiques, économiques, politiques, et culturels différents. Depuis peu, les anthropologues se tournent vers la télévision et les médias de masse comme objets d'étude, bien que la présence des médias ne soit pas récente. La télévision et les médias de masse influencent notre vie quotidienne depuis bien des années et même dans les sociétés les plus lointaines, comme c'est le cas pour les peuples indigènes du Mexique, les aborigènes en Australie ou les peuples autochtones en Amazonie, qui s'approprient les technologies pour s'auto-représenter en images afin de prendre le pouvoir (empowerment) contre l'impérialisme culturel et économique occidental ${ }^{3}$.

La mondialisation de l'image permet davantage l'existence d'un monde fluide en perpétuel changement et tissé par les interrelations, les échanges et les interactions culturels dans un monde où les frontières entre les cultures deviennent poreuses et tissent des liens qui donnent lieu à l'émergence de "modernités parallèles ". L'anthropologie des médias rejette la dichotomie entre la tradition et la modernité basée sur la reconnaissance que nous sommes tous "modernes" ou, pour reprendre Bruno Latour (1995), que nous ne l'avons jamais été, si l'on considère que la modernité est une fiction construite par la science occidentale. De ce fait, l'accent mis sur les processus de médiation, renforcés par la mondialisation, annonce la mort du concept de "culture " comme un ensemble partagé par tous les membres d'une société donnée. Les anthropologues se montrent en effet de plus en plus méfiants quant à l'étude des cultures comme des ensembles, pour éviter les erreurs commises par les primordialistes qui tendaient à homogénéiser et exoticiser les personnes - surtout concernant les peuples des sociétés qualifiés "traditionnelles » - et à nier leur historicité. La "culture ", entendue comme un monolithe, est mise à l'écart pour explorer les différences internes, les identités, modernités et subjectivités qui se produisent à partir des transformations historiques, évoquant à la fois des sentiments d'appartenance, et le pouvoir de s'auto-représenter, de se "faire " (making) une ou des cultures.

Ainsi, la présence de la télévision ne relève pas uniquement de l'ordre de la réception (passive) des images projetées. L'exemple de la coopération entre les Nahuas et Teenek de la Huastèque et une émission télévisée sur le Mexique rural illustrent une deuxième modalité des processus de médiation entre culture et mondialisation. En 2002, les anciens Teenek et Nahuas m'ont invitée à assister à la première célébration de leur Cérémonie du Feu Nouveau dans une ville municipale. Ils avaient alors pris la décision de célébrer cette cérémonie annuelle sur la place municipale du chef-lieu de leur commune ainsi que devant les caméras de la chaîne TV Azteca pour une émission culturelle. Selon leurs témoignages, les aînés voulaient saisir cette occasion pour montrer avec dignité leurs coutumes à un public à majorité métisse. La cérémonie durait plusieurs heures pendant lesquelles les spécialistes Teenek ont présenté des offrandes à la terre, au soleil, au vent, à la pluie, et au feu. Le présentateur, un grand Mexicain blond aux yeux bleus, m'a expliqué qu'il souhaitait rendre visibles ces sociétés longuement condamnées à l'invisibilité par les médias. Deux mois après l'événement, une synthèse de la cérémonie était diffusée sur la chaîne nationale Televisa dans une édition spéciale de l'émission MeridianoX sur la semaine de Pâques ( $L a$ Semana Santa de la Huasteca»). Cet exemple de l'appropriation de la télévision par les peuples indigènes Nahuas et Teenek pendant la cérémonie du feu démontre que les médias peuvent servir d'espace dynamique des «luttes sur les modes de représentation» (Spitulnik 1993).

L'anthropologie des médias se situe au fondement même d'une anthropologie du monde contemporain qui nous approche de la contemporanéité (coevalness) entre l'anthropologue et son objet d'étude. Quand je me suis rendue au Mexique pour faire mon premier terrain en 1996, je me suis rendu compte du fait que l'anthropologue « ne peut pas uniquement 
travailler avec des petits groupes, qu'avec des interlocuteurs singuliers", mais que l'on doit tenir compte du fait que chaque interlocuteur est " au croisement de différent mondes et différentes vies » (Augé 1994 : 170). Marc Augé souligne que l'heure est venue d'améliorer notre regard afin de créer une "contemporanéité effective et vécue " et il nous offre des conseils méthodologiques à cet égard, par exemple en s'intéressant davantage aux phénomènes sociaux que sont l'urbanisation, le développement, et la technologie (Augé 1994 : 80). Ce changement de perspective en anthropologie s'explique en partie par le développement récent dans la discipline d'une anthropologie "après le colonialisme " ou "postcoloniale » (Abu-Lughod 2002 ; Appadurai 1996). Il n'est guère surprenant que, dès lors, de nouvelles problématiques et de nouvelles questions émergent pour remplacer les terrains classiques $^{4}$.

\section{Les médias et l'imaginaire national}

Depuis l'article de Benedict Anderson sur la nation comme une "communauté imaginée", les médias ont été analysés comme véhicules par excellence pour projeter des narrations de la nation et pour forger les identités nationales (Anderson 1983 ; Faris 1996 ; Lutz and Collins 1993 ; Shohat and Stam 1994, 2003). Edward Saïd souligne qu'un des débats contemporains sur les résidus de l'impérialisme est la représentation des autochtones dans les médias de l'Occident, mettant en avant dans ce débat non seulement ce qui se dit mais "comment, par qui, où, et pour qui » (1993:21). Ce genre de "visualisme colonial ", pour reprendre la notion de Terry Smith (1998), a donné lieu à de nombreuses études sur les régimes visuels de la colonisation, reprises par les critiques postcoloniales des modes de représentation et de l'autorité ethnographique en anthropologie (voir par exemple McClintock 1998 ; Mitchell 1998).

La critique de cet " orientalisme visuel " dans la reproduction d'un imaginaire national par la transmission des images conforte l'idée de Roland Barthes (1998) concernant la "rhétorique de l'image ", qui devient "support de sens" par le biais de la représentation. Si on prend l'exemple de l'image publicitaire de Barthes, on trouve effectivement l'intention de la signification suggérée de l'image. Avec la télévision, l'image peut également suggérer une signification précise. Pourtant, le contexte de l'émission et de la réception des images projetées offre de multiples sens possibles générés par l'expérience vécue de l'image. Ainsi, nous considérons que la projection d'un imaginaire national n'a pas nécessairement les effets souhaités. Toutefois, la réception et la transformation en images vécues à travers les processus de médiation permettent des significations multiples qui donnent lieu à une hétérogénéité de modernités (Abu-Lughod 2002 ; Wilk 2002).

$\mathrm{Au}$ Mexique, les deux grandes chaînes nationales ciblent leurs audiences par région : en général parlant, la chaîne Televisa vise le grand public des centres urbains, et la chaîne TV Azteca s'adresse aux ruraux. Ainsi, Televisa émet davantage d'émissions "culturelles " sur l'histoire, l'art, et l'architecture tandis que TV
Azteca s'adresse à un public plus populaire avec les telenovelas qui diffusent des images d'histoires des rancheros (familles riches de la campagne) et leurs interactions avec les petits paysans ainsi que les images d'histoires des ruraux qui arrivent en ville. Or, bien que les textes culturels diffusés par les médias de masse et la télévision possèdent toujours un pouvoir indéniable sur leurs audiences, il ne s'ensuit pas automatiquement que les audiences soient des récepteurs passifs, qui seraient modelés et façonnés par les messages principaux contenus dans la projection des médias. Par conséquent, la médiation de l'image se met en place par un processus de va-et-vient entre le public ciblé et le public réel, entre la signification suggérée et le sens vécu (et interprété) par le public.

Les analyses récentes des relations entre les médias et le nationalisme à l'heure de la mondialisation tendent à diminuer l'importance du pouvoir des médias à contrôler les citoyens, tout en mettant en avant les visions émergentes de différenciation et de contestation, notamment, par rapport aux appropriations stratégiques des images projetées, voir l'appropriation des outils médiatiques par les groupes marginalisés (McLagan 2002 ; Prins 2002 ; Turner 1992, 2002). L'anthropologue indien et américain Arjun Appadurai (1996) nous invite à penser les conséquences culturelles de la mondialisation, notamment, les conséquences de la médiatisation des cultures et la création des paysages médiatiques (mediascapes) à travers lesquels l'imagination devient une force sociale qui sert à remettre en cause la puissance et l'omniprésence de l'Occident et de l'État-nation.

$\mathrm{Au}$ Mexique, l'adoption des lois du marché néoliberal bénéficie aux chaînes privées, telles que Televisa et TV Azteca (appartenant aux familles les plus riches du Mexique), qui diversifient peu à peu ces «paysages médiatiques" afin de toucher les nouvelles populations de consommateurs, dont les ruraux. $\mathrm{Au}$ lieu de concevoir la médiatisation d'une culture nationale suggérée par les images transmises par la télévision, nous avons affaire à un tissage émergent de «cultures » variées.

Ceci semble se produire avec le succès récent de l'émission La Academia, l'équivalent de la Star Academy française au Mexique. Le 20 mars 2004, lors de la deuxième génération de La Academia, l'élève Erasmo Gonzales monte sur scène pour chanter le tube "La Manzanita» du groupe légendaire Los Tigres del Norte. Il est loué par le public qui doute pourtant de ses capacités à gagner du fait de son physique et de ses origines indigènes. En effet, Erasmo, un maestro bilingue (instituteur dans les écoles maternelles bilingues où l'on enseigne en langue indigène et en espagnol) est d'origine nahua. Il est plutôt petit, avec un visage rond, un grand sourire, une moustache, la peau matte et des cheveux noirs hérissés, un physique associé dans l'imaginaire populaire aux gens pauvres qui vivent en province.

La présence d'Erasmo est pertinente dans le contexte de la représentation car la première génération de La Academia (composée principalement par des Mexicains «blancs») avait suscité de nombreuses critiques du public, qui considérait que la composition du groupe 
d'élèves ne reflétait pas la réalité de la nation. En revanche, pendant la deuxième génération, la sélection des élèves lors du casting avait permis pour la première fois la présence de Mexicains ayant une apparence physique jusque là rarement représentée à la télévision mexicaine, où dominent les grands blonds de peau blanche aux yeux bleus. À La Academia, l'inclusion des élèves d'origine indigène, et les programmes «culturels " démontrent une transformation quant à la visibilité des groupes marginaux qui commencent à se manifester en tant que citoyens mexicains. Quatre mois plus tard La Academia entre véritablement dans la "réalité " de la démographie mexicaine au moment où le public ayant le pouvoir de l'ultime décision dans ce concours - vote pour Erasmo, qui est désormais le gagnant de La Academia 2004.

Depuis les années 90 , on note l'émergence des productions des médias indépendants des "peuples sans histoire» (Wolf 1982), comme le Chiapas Media Project au Mexique. Les médias indépendants sont souvent d'abord financés avec l'aide d'ONGs ou obtiennent des fonds par d'autres organismes nationaux ou internationaux. Par ces productions indépendantes, les peuples indigènes, qui dès lors deviennent producteurs et contrôlent leurs propres images et représentations visuelles, commencent à affirmer publiquement leur hétérogénéité et leur historicité en créant de nouvelles alliances à travers les frontières locales et nationales, et à travers les barrières ethniques et linguistiques. Bien que les productions indépendantes restent encore rares au Mexique, surtout faute de financements, il y a par ailleurs des coalitions entre les personnes rurales et les chaînes de télévision comme le montre l'exemple la cérémonie du feu. Par conséquent, les médias, autrefois conçus comme un moyen de domination et de contrôle d'un imaginaire homogène national, acquièrent de nouveaux degrés de significations pour les peuples indigènes et d'autres groupes marginalisés, car ce sont eux qui s'approprient ces nouvelles technologies pour en faire les « armes des faibles » au sens de James Scott (1985).

Les exemples visent à illustrer quatre modalités principales de la médiation qui donnent lieu à ce que j'ai appelé des "imaginaires alternatifs ». Il y a d'abord l'interprétation des images comme des représentations (étrangères) et leur transformation en expériences vécues dans la vie quotidienne des personnes, comme l'a démontré le premier exemple, l'avènement de la télévision dans les villages de la Huastèque. Puis la nouvelle présence des ruraux et des peuples indigènes (ou d'autres groupes auparavant "invisibles") dans les reality show comme l'émission La Academia. Il y a ensuite l'appropriation des chaînes télévisées privées par des gens locaux avec une participation "stratégique", qui devient une politique de visibilité, comme c'était le cas lors de l'émission culturelle la Semana de la Huasteca. Finalement, la production indépendante de programmes de télévision et de films par les groupes marginalisés comme le Chiapas Media Project sert encore à diversifier les paysages médiatiques du Mexique et apporte une véritable dimension politique, dans le sens d'un "anti-colonialisme visuel». Bien entendu, chaque modalité se produit de différentes manières en fonction du contexte.
Comme le soulignent les exemples, les forces de la mondialisation ont diversifié ce que l'on entendait autrefois par "la culture ", qui dès lors n'est plus un ensemble homogène mais un tissage de subjectivités médiatisées et d'imaginaires alternatifs ${ }^{5}$

\section{De la provincialisation du Mexique}

Malgré les définitions multiples que l'on attribue à la mondialisation, qui reste un terme contesté et difficile à saisir, on peut avancer que la mondialisation reflète deux tendances principales, l'amplification de la communication à niveau global et l'intensification du marché. En ce sens, la mondialisation représente une nouvelle variation de la fameuse phrase de Marx et Engels qui caractérise le désenchantement de la condition de l'humain moderne : "tout ce qui est solide fond dans l'air ».

Cependant, si ce sont les circonstances de la mondialisation qui ont entrainé une mise en perspective des anciennes dichotomies entre modernité et tradition, il ne s'agit pas seulement d'une nouvelle variation du désenchantement moderniste, si l'on considère que les choses n'étaient pas aussi "solides» qu'elles apparaissaient (Mazarrella 2004). La dynamique de la mondialisation est un double processus, la particularisation de l'universel et l'universalisation du particulier. Cependant, il importe de considérer les relations structurelles qui continuent à exister dans le domaine du politique, de l'économique, et du culturel. Enrique Dussel (1998) nous rappelle par ailleurs que la "crise de la modernité " (déjà abordée par Nietzsche et Heidegger) est une crise typiquement européenne et relève par excellence de l'ethnocentrisme. Avec la mondialisation de l'image, nous sommes alors confrontés à un nouveau registre de subtilités et de nuances de la mondialisation, illustré à travers les exemples des différentes modalités de la médiation.

La mondialisation de l'image qui a animé cette réflexion sur la télévision au Mexique démontre que la mondialisation peut acquérir des connotations négatives et positives. La télévision reste souvent associée à la domination par l'imaginaire de la modernité, similaire à "l'imaginaire du développement " (Abu-Lughod 2005). Ainsi, l'invasion des images télévisées dans les villages indigènes peut prendre le sens « d'un néocolonialisme visuel» qui peut en effet engendrer une homogénéisation de la diversité des cultures par une certaine "culture globale". En ce sens, l'image d'un "Mexique silencieux " proposé par Claudio Lomnitz (2001) s'affirmerait par l'absence des voix des peuples indigènes et des ruraux dans les médias. Par ailleurs, le néocolonialisme visuel pourrait également conduire à ce que j'appellerai un "Mexique invisible" avec l'absence d'images d'autoreprésentation des peuples indigènes et des ruraux à l'écran.

Ma réflexion sur la mondialisation de l'image et les modalités d'appropriation des technologies (occidentales) démontre pourtant que la télévision peut également servir de source pour l'affirmation, la visibilité, l'auto-représentation, voire la «libération " des ruraux et des peuples 
autochtones, qui historiquement étaient privés d'accès aux technologies visuelles. J'ai montré qu'ils se manifestent dès lors en tant que peuples autochtones et citoyens mexicains en tissant des alliances avec des agents extérieurs (ONG, gouvernements, organisations internationales, chaînes de télévision), et deviennent parfois les producteurs de leurs propres images. C'est ainsi, me semble-t-il, que la mondialisation de l'image introduit au Mexique une «provincialisation" du pays à travers une nouvelle visibilité à la province, un Mexique autrefois "invisible " sur les écrans. Dès lors, le Mexique est mis en scène par les ruraux et des peuples marginalisés qui vivent dans la périphérie ${ }^{6}$. De ce fait, la mondialisation de l'image semble permettre une diversification des paysages de médias par la présence de modernités émergentes et l'autoreprésentation des images alternatives de la nation.

\section{Kristina TIEDJE}

\section{Notes}

J'ai changé les noms pour protéger les identités de mes interlocutrices.

Les fouilles archéologiques dans la région se limitent à quelques lieux représentatifs de la civilisation huastèque (Ekholm 1944, Stresser-Péan 1971).

Depuis les années 70, les aborigènes d'Australie et les peuples autochtones d'Amérique Latine ont développé de nouvelles formes visuelles en adaptant les technologies de la vidéo, du film, et de la télévision à des fins politiques et expressives (Ginsburg 1994 ; Turner 1992 ; Smith 2002).

4 Par exemple, au lieu d'étudier l'organisation politique d'une communauté donnée, les anthropologues s'interrogent sur les constructions identitaires, sur les politiques culturelles et les interactions entre les organisations locales, nationales et transnationales. De même, les études de genre prennent le relais des études des systèmes de parenté, surtout avec l'émergence de nouvelles technologies de reproduction, et l'intérêt porté aux faits relevant du religieux, aux nouvelles religions et aux fondamentalismes succède à l'analyse des cosmologies.

$5 \quad$ Néanmoins, il importe de noter que la production industrielle des telenovelas projetant d'autres idéaux dans les communautés des ruraux et des peuples autochtones peut également générer des conflits entre les générations. Les personnes âgées jugent le style vestimentaire et le comportement des femmes inappropriées.

6 Je m’inspire des écrits de Chakrabarty (2000) sur la "provincialisation de l’Europe » à l'époque postcoloniale.

\section{RÉFÉRENCES BIBLIOGRAPHIQUES}

- Abu-Lughod L., 2002, Egyptian Melodrama-Technology of the Modern Subject ? In : Media Worlds. F. D. Ginsburg, Lila Abu-Lughod, and Brian Larkin, ed. p.p. 115-133. Berkeley, CA : University of California Press.

- $\quad$ Abu-Lughod L., 2002, Dramas of Nationhood. The Politics of Television in Egypt. Chicago : University of Chicago Press.

- $\quad$ ANDERSON B., 1983, Imagined Communities. New York : Verso.

- $\quad$ Appadurai A., 1996, Modernity at Large. Cultural Dimensions of Globalization. Minneapolis, MN : University of Minnesota Press.

- Auge M., 1994, Pour une anthropologie des mondes contemporains. Paris: Champs, Flammarion.

- $\quad$ BARTHes R., 1998, Rhetoric of the Image. In : The Visual Culture Reader. N. Mirzoeff, Ed. p.p. 135-138. London and New York : Routledge.

- Bonfil Batalla G., 1987, México profundo. Una civilización negada. Mexico City : Grijalbo.

- Chakrabarty D., 2000, Provincializing Europe. Postcolonial Thought and Historical Difference. Princeton and Oxford : Princeton University Press.

- De Janvry A., Sadoulet E., Davis B., and Gordillo de Anda G., 1996, Ejido Sector Reforms : From Land Reform to Rural Development. In : Reforming Mexico's Agrarian Reform. L. Randall, ed. p.p. 71-106. London : M.E. Sharpe Inc.

- Dussel E., 1998, Beyond Eurocentrism : The World-System and the Limits of Modernity. In : The Cultures of Globalization. F. J. a. M. Miyoshi, Ed. p.p. 3-31. Durham and London : Duke.

- Ekholm G. F., 1944, Excavations at Tampico and Pánuco in the Huasteca, Mexico. Anthropological Papers of the American Museum of Natural History 38 :321-507.

- FARIS J. C., 1996, Navajo and Photography: A Critical History of the Representation of an American People. Albuquerque : University of New Mexico Press. 
- $\quad$ Latour B., 1995, Nous n'avons jamais été modernes : Essai d'anthropologie symétrique. Paris : La Découverte.

- $\quad$ Lomnitz C., 2001, Deep Mexico, Silent Mexico. An Anthropology of Nationalism. London and Minneapolis : University of Minnesota Press.

- $\quad$ Lutz C. and Collins J., 1993, Reading National Geographic. Chicago : University of Chicago Press.

- Mazarella W., 2004, Culture, Globalization, Mediation. Annual Review of Anthropology $33: 345-67$.

- McClintock A., 1998, Soft-Soaping Empire : Commodity Racism and Imperial Advertising. In: The Visual Culture Reader. N. Mirzoeff, Ed., p.p. 506-518. London and New York: Routledge.

- MCLagan M., 2002, Spectacles of Difference : Cultural Activism and the Mass Mediation of Tibet. In : Media Worlds. F. D. Ginsburg, Lila Abu-Lughod, and Brian Larkin, Ed., p.p. 90-111. Berkeley : University of California Press.

- Mitchell T., 1998, Orientalism and the Exhibitionary Order. In : The Visual Culture Reader. N. Mirzoeff, Ed., p.p. 495-505. London and New York : Routledge

- PRins H. E. L., 2002, Visual Media and the Primitivist Complex : Colonial Fantasies, Indigenous Imagination, and Advocacy in North America. In : Media Worlds. F. D. Ginsburg, Lila Abu-Lughod, and Brian Larkin, Ed., p.p. 58-74. Berkeley, CA : University of California Press.

- $\quad$ SASsen S., 1998, Globalization and Its Discontents. New York : The New York Press.

- $\quad$ SAÏD E., 1993, Culture and Imperialism. New York : Vintage Books.

- $\quad$ ScotT J., 1985, Weapons of the Weak. New Haven, CT : Yale University Press.

- $\quad$ Sectur M., 2004, Estudio de Viabilidad. Vol. 2005 : Secretaría de Turismo de México.

- Shohat E. and Stam R., 1994, Unthinking Eurocentrism : Multiculturalism and the Media. London and New York : Routledge.

- Shohat E. and Stam R., 2003, The Imperial Imagery. In : The Anthropology of Media. R.R. Wilk and K. Askew, Ed. Malden : Blackwell.

- $\quad$ Smith T., 2002, Visual Regimes of Colonization : Aboriginal Seeing and European Vision in Australia. In: The Visual Culture Reader. N. Mirzoeff, Ed., p.p. 483-494. New York: Routledge.

- $\quad$ SpitulniK D., 1993, Anthropology and Mass Media. Annual Review of Anthropology 22 : 293315.

- Stephen L., 2002, ¡Zapata Lives! Histories and Cultural Politics in Southern Mexico. Berkeley, CA : University of California Press.

- Stresser-Pean G., 1971, Ancient Sources on the Huasteca. In : Handbook of Middle American Indians. Vol. 10-11. G. F. Ekholm, ed. Austin, TX : University of Texas Press.

- TIEDJE K., 2002, Gender and Ethnic Identity in Rural Grassroots Development : An Outlook from the Huasteca. Urban Anthropology and Studies of Cultural Systems and World Economic Development 31(3-4):261-316.

- Turner T., 1992, Defiant Images : The Kayapo Appropriation of Video. Anthropology Today $8(6): 5-16$.

- Turner T., 2002, Representation, Politics, and Cultural Imagination in Indigenous Video : General Points and Kayao Examples. In : Media Worlds. L. Abu-Lughod, Faye D. Ginsburg, and Brian Larkin, Ed., p.p. 75-89. Berkeley, CA : University of California Press.

- Wilk R. R., 2002, Television, Time, and the National Imagery in Belize. In : Media Worlds. L. Abu-Lughod, Faye D. Ginsburg, and Brian Larkin, Ed., p.p. 171-189. Berkeley, CA : University of California Press.

- Wolf E., 1982, Europe and The People Without History. Berkeley, CA : University of California Press. 